\title{
Sirolimus in Kidney Transplantation From Marginal Donors
}

\author{
F. Pisani, O. Buonomo, G. laria, B. Iorio, A. Rizzello, S. Pollicita, L. De Luca, M. Valeri, V. Boffo, \\ A. Famulari, and C.U. Casciani
}

\begin{abstract}
Nephrotoxicity caused by calcineurin inhibitors can lead to either delayed graft function or long-term decline of renal function after kidney transplantation. Therefore, recipients of renal transplants from marginal donors require non-nephrotoxic immunosuppression. Eighteen patients received kidney transplants from marginal donors, with a calcineurin inhibitor-free immunosuppressive regimen, based on basiliximab, mycophenolate mofetil, steroids, and sirolimus. Renal graft biopsy was performed in all cases before surgery. Mean follow-up was 11.8 months. We report immediate renal function in 9 patients, delayed graft function in 5 and acute tubular necrosis in 4 patients. One patient was successfully treated for biopsy-proven acute rejection. Hypercholesterolemia and hypertriglyceridemia were the most common adverse effects $(n=13)$ associated with arthralgia $(n=2)$ and thrombocytopenia $(n=2)$. Five patients underwent a switch to tacrolimus, due to sirolimus-induced side effects. Immunosuppression without the use of calcineurin inhibitors is a safe and effective regimen in kidney transplantation, although sirolimus-related side effects still represent a morbidity factor in these patients.
\end{abstract}

$\mathrm{T}$ HE SCARCITY of organs suitable for transplantation has led kidney transplant teams to expand the pool with the use of elderly or marginal donors. ${ }^{1}$ In such settings, recovery of renal graft function may be negatively influenced by immunosuppressive therapy. Calcineurin inhibitors are nephrotoxic drugs that cause acute as well as chronic renal injuries. Acute nephrotoxicity that occurs in the early period after transplantation may lead to a higher rate of dialysis. Chronic nephrotoxicity may result in graft loss. ${ }^{2}$

\section{PATIENTS AND METHODS}

In June 2001, our transplant center started a protocol based on calcineurin inhibitor-free immunosuppressive for marginal kidney transplant donors. Since March 2003, 18 patients were enrolled in the study. Marginal donors were defined as: older than 60 years of age $(\mathrm{n}=8$; mean age, 67.4 years; \pm 5.2$)$, history of hypertension ( $\mathrm{n}$ $=6$ ), length of stay in intensive care unit more than 10 days, and hypotension $(n=4)$. Donor renal biopsies, which were performed in all cases, showed a score between 2 and $3 .^{2}$ Baseline recipient characteristics were as follows: mean age, 52 years $( \pm 11)$; men (n $=13$ ); and women $(\mathrm{n}=5)$. Moreover, 3 patients had a previous renal transplant. The immunosuppressive protocol used induction with basiliximab $(20 \mathrm{mg}$ on day 0 and 4$)$, and maintenance with prednisone (20 mg tapered to $5 \mathrm{mg}$ on month 6 ), and mycophenolate mofetil (MMF) (1500 mg divided into 2 daily doses). Sirolimus was started 12 hours after transplantation with a loading dose of 6 $\mathrm{mg}$ tapered to $3 \mathrm{mg}$ on day 3 afterwards; sirolimus doses were adjusted to maintain 24-hour trough levels at $7-12 \mathrm{ng} / \mathrm{mL}$ for 6 months and 5-10 $\mathrm{ng} / \mathrm{mL}$ thereafter.

\section{RESULTS}

Mean follow-up was 11.8 months $( \pm 7.6)$. Immediate graft function was achieved in 9 patients with no need for dialysis, 5 patients had delayed graft function (DGF), and 4 patients had acute tabular necrosis (ATN). However, all patients but the 1 who lost the graft had a good recovery with a mean creatinine level of $1.8 \mathrm{mg} / \mathrm{dL}( \pm 0.8)$ after 1 month. One patient was treated for a biopsy-proven acute rejection episode at 5 days after transplantation with steroids and a switch from sirolimus to tacrolimus.

Sirolimus-related side effects were observed in 13 patients; hypercholesterolemia and hypertriglyceridemia were not related to high trough levels of sirolimus. Associated with dyslipemie were arthralgia $(\mathrm{n}=2)$ and mild thrombocytopenia $(n=2)$. Our approach to these side effects was to

From the Clinica Chirurgica, Università di Roma Tor Vergata, and Dip.di scienze Chirurgiche UO Trapianti Università di L'Aquila, Italy.

Address reprint requests to F. Pisani, Clinica Chirurgica, Ospedale S. Eugenio, Piazzale Umanesimo 10, 00144 Roma, Italy.

0041-1345/04/\$-see front matter doi:10.1016/j.transproceed.2004.02.011 
reduce sirolimus exposure to a tough level of $5 \mathrm{ng} / \mathrm{mL}$ and 10 increase MMF to $2000 \mathrm{mg} / \mathrm{d}$, and thereafter to carefully administrate statins with routine examination of CPK. The switch from sirolimus to tacrolimus was performed in 4 patients due to hypercholesterolemia and mild increases in serum creatinine levels.

\section{CONCLUSIONS}

It has been demonstrated that avoidance of calcineurin inhibitors may be achieved with low acute graft rejection episodes using sirolimus. ${ }^{1-5}$ This strategy can be useful to expand the donor pool with the use of marginal kidneys, whose function may be easy affected by calcineurin inhibitor nephrotoxicity. However, proper matching between the recipient and the immunosuppressive regimen is recommended due to sirolimus-related side effects. In fact, both hypercholesterolemia and hypertriglyceridemia are common findings in elderly patients, who are generally allocated a marginal graft. Therefore, our finding suggest the value of avoiding calcineurin inhibitors by the use of sirolimus with basiliximab, steroids, and MMF in marginal kidneys. If sirolimus-related side effects appear during follow-up, it is recommended to switch to tacrolimus.

\section{REFERENCES}

1. Donati D, Ambrosiani A, Marconi A, et al: Calcineurin inhibitor free immunosuppressive regimen for marginal donors/ recipients of kidney transplantation. Transplant Proc 34:1678, 2002

2. Pescovitz MD, Giovani M: Sirolimus and mycophenolate mofetil for calcineurin-free immunosuppression in renal transplant recipients. Am J Kidney Dis 38(4 suppl 2):S16, 2001

3. Remuzzi G, Gringo J, Ruggenenti P, et al: Early experience with dual kidney transplantation in adults using expanded donor criteria. J Am Soc Nephrol 10:2591, 1999

4. Flechner SM, Goldfarb D, Modlin C, et al: Kidney transplantation without calcineurin inhibitor drugs: a prospective, randomized trial of sirolimus versus cyclosporine. Transplantation 74:1070, 2002

5. Stegall MD, Larson TS, Prieto M, et al: Kidney transplantation without calcineurin inhibitors using sirolimus. Transplant Proc 35(3 suppl):S125, 2003 